\title{
Objective Evaluation Of On-Eye Optical Quality Of Daily Disposable Silicone Hydrogel Contact Lens With Internal Wetting Agents
}

This article was published in the following Dove Press journal:

Clinical Ophthalmology

\author{
Shizuka Koh (1) ${ }^{1,2}$ \\ Kiyoshi Watanabe ${ }^{3}$ \\ Kohji Nishida ${ }^{2}$ \\ 'Department of Innovative Visual \\ Science, Osaka University Graduate \\ School of Medicine, Osaka, Japan; \\ ${ }^{2}$ Department of Ophthalmology, Osaka \\ University Graduate School of Medicine, \\ Osaka, Japan; ${ }^{3}$ Watanabe Eye Clinic, \\ Osaka, Japan
}

Purpose: To investigate the on-eye optical quality of a daily disposable silicone hydrogel contact lens with internal wetting agent.

Patients and methods: Study subjects were daily disposable soft contact lens wearers $(\mathrm{N}=20)$ who were instructed to wear daily disposable hydrogel etafilcon A lens (without added wetting agent) or daily disposable silicone hydrogel senofilcon A lens (with an internal wetting agent), for a week. Subjects wore their respective lenses bilaterally and disposed of the pair of lenses daily. At the end of the 1-week test-wear period, the on-eye visual performances of the lens and the ocular surfaces were evaluated. A wavefront sensor measured sequential ocular higher-order aberrations (HOAs) for $10 \mathrm{~s}$ after the blink. The aberration data were analyzed in the central 4-mm diameter up to the sixth-order Zernike polynomials. Total HOAs, fluctuation index (FI), and stability index (SI) of the total HOAs over time were compared between the two lenses. Ocular surface evaluation with fluorescein was performed following the wavefront measurement.

Results: The senofilcon A lens had significantly lower average total HOAs, FI, and SI ( $p<0.001, p=0.001, p=0.007$, respectively) than the etafilcon A lens. After 1-week wear of each lens, corneal staining was observed in eight subjects (40\%) with the etafilcon A lens and in two subjects $(10 \%)$ with the senofilcon A lens. The senofilcon A lens had significantly lower incidence of corneal staining than the etafilcon A lens $(p=0.041)$.

Conclusion: Quantitative sequential measurement of HOAs objectively showed the possibility of better and more stable optical quality with silicone hydrogel daily disposable contact lens with the internal wetting agent than with hydrogel lens without the added wetting agent. Keywords: visual performance, quantitative assessment, surface lens wettability

\section{Introduction}

Silicone hydrogel (SiHy) was commercially introduced as a contact lens (CL) material in the late $1990 \mathrm{~s},{ }^{1-3}$ and the prescription rate of SiHy CLs has since been increasing worldwide. First-generation SiHy CLs for extended wear have attempted to address the issue of oxygen permeability during continuous wear. However, first-generation SiHy CLs had lower water content and higher modulus than the hydrogel lenses, making them stiffer and sometimes causing mechanical complications owing to non-optimal fitting. Recent developments and innovations aiming to minimize mechanical issues causing discomfort and adverse events with SiHy CLs have led to the design of SiHy daily disposable CLs that combine the advantages of both the SiHy material and the daily disposable modality. Improved
Correspondence: Shizuka Koh

Department Of Innovative Visual Science, Osaka University Graduate School Of

Medicine, Room E7, 2-2 Yamadaoka,

Osaka 565-087I, Japan

Tel +8I-6-6879-3456

Fax +8I-6-6879-3458

Email skoh@ophthal.med.osaka-u.ac.jp 
safety outcomes of daily disposable CLs were reported by recent studies, ${ }^{4}$ though neither SiHy nor hydrogel had shown superiority in terms of comfort, with adverse event rates being low for both materials as daily disposable CLs. ${ }^{5}$

A common CL discomfort reported by soft contact lens (SCL) wearers is visual disturbance manifesting as blurring or fluctuating vision. ${ }^{6}$ Previous reports have studied the oneye optical quality of hydrogel daily disposal lens. ${ }^{7,8} \mathrm{We}$ previously measured the ocular higher-order aberrations (HOAs) in eyes with hydrogel daily disposal lens and demonstrated an increase in postblink HOAs after the blink. ${ }^{7}$ A study using dynamic area high-speed videokeratoscopy and lateral shearing interferometry showed that there was a significant decrease in the prelens tear film quality with respect to the baseline precorneal tear film quality with daily hydrogel CLs over one day of use. ${ }^{8}$ These quantitative objective measurements suggested that surface wetting of a $\mathrm{CL}$ is an important factor that influences optical quality. The material and water content of CLs may affect surface lens wettability and its interactions with the ocular tear film and ocular surface. Recently, several studies ${ }^{9-13}$ have reported the clinical performance of SiHy daily disposable CLs; however, little is known about the on-eye optical quality of the SiHy daily disposable CLs. ${ }^{14}$

The purpose of this study was to investigate the optical quality of a SiHy daily disposable CL with internal wetting agent using quantitative sequential wavefront measurement in a clinical setting, which would provide new insights into selection of CLs from the viewpoint of visual performance.

\section{Materials And Methods}

This prospective study was reviewed and approved by our institutional review board. The study adhered to the tenets of the Declaration of Helsinki. All participants provided written informed consent after receiving an explanation of the nature and possible consequences of the study.

Twenty experienced SCL wearers between 20 and 40 years of age, who had been wearing spherical disposable SCLs (either SiHy or hydrogel) in both eyes and were able to wear CLs more than $12 \mathrm{hrs}$ per day, were enrolled in the study. Exclusion criteria included the following conditions: extended wear of SCLs, wear of toric or multifocal SCL, having clinically significant anterior segment abnormalities or ocular and systemic diseases that would preclude SCL wear, habitual Snellen visual acuity of less than 20/30 in either eye, and being pregnant or lactating. A daily disposablehydrogel etafilcon A lens without the added wetting agent (1-Day ACUVUE; Johnson \& Johnson K.K. Vision Care Company, Tokyo, Japan) and a daily disposable SiHy senofilcon A lens with pure high molecular weight polyvinyl pyrrolidone (1-Day ACUVUE OASYS; Johnson \& Johnson K.K. Vision Care Company) were fit to the eye of each subject. Details of both products are shown in Table 1.

Before the study, it was confirmed that both types of lenses would properly fit the eye of each subject. Each subject wore both types of test lenses in a randomized order. That is, subjects wore either etafilcon A or senofilcon A lens for 1 week. Both lenses were fit bilaterally as a daily wear with daily disposable modality. At the end of the first week, the on-eye visual performance of the lenses and the ocular surfaces were evaluated. Then, each subject wore the other type of CL for 1 week, following which the same measurements were taken. One eye of each subject was chosen in randomized manner. The measurements were performed between 6:00 and 8:00 P.M. in a room where the temperature and humidity were controlled at $22^{\circ}$ $\mathrm{C} \pm 2{ }^{\circ} \mathrm{C}$ and $40 \% \pm 5 \%$.

Table I Details Of Tested Contact Lenses

\begin{tabular}{|l|l|l|}
\hline Brand Name & I-Day ACUVUE & \\
\hline Manufacturer & Johnson \& Johnson Vision Care, Inc. & ACUVUE $^{\circledR}$ OASYS $^{\circledR}$ I-Day With HydraLuxe \\
Material & Technology \\
Internal wetting agent & None & Senofilcon A \\
FDA classification & Group IV & High molecular weight polyvinyl pyrrolidone \\
Water content (\%) & $58 \%$ & Group I \\
Base curve $(\mathrm{mm})$ & $8.5 / 9.0 \mathrm{~mm}$ & $38 \%$ \\
Diameter $(\mathrm{mm})$ & $14.2 \mathrm{~mm}$ & $8.5 / 9.0 \mathrm{~mm}$ \\
Center thickness $(\mathrm{mm})$ & $0.07 \mathrm{~mm}(-3.00 \mathrm{D})$ & $14.3 \mathrm{~mm}$ \\
Method of manufacture & Stabilized soft molding & $0.085 \mathrm{~mm}(-3.00 \mathrm{D})$ \\
\hline
\end{tabular}


The ocular HOAs were measured continuously every second for $10 \mathrm{~s}$ after a blink, using a Hartmann-Shack aberrometer (KR-1W; Topcon Corporation, Tokyo, Japan), through a natural pupil. The wavefront data were analyzed quantitatively in a central 4-mm area of the pupil up to the sixth order by expanding the set of Zernike polynomials. From the Zernike coefficients, the root mean square was calculated to represent the wavefront aberrations. S3, S4, S5, and S6 are the root mean squares of the third-, fourth-, fifth-, and sixth-order Zernike coefficients, respectively. The total HOAs were computed by adding all the components (S3+S4+S5+S6) ${ }^{15}$ In accordance with previous studies, ${ }^{7,16}$ two more quantitative indices, the fluctuation index (FI) and the stability index (SI) of the total HOAs, were used to indicate the sequential change in HOAs. FI is the average standard deviation of the total HOAs between blinks. SI is the slope of the linear regression line of the total HOAs between blinks.

Following the wavefront measurement, ocular surface examination with slit-lamp was performed by removing the CLs from the eyes and applying fluorescein to the cornea, after CL fitting confirmation. The presence or absence of fluorescein corneal staining was evaluated.

Statistical analysis was performed using SigmaPlot version 14.0 for Windows (Systat Software Inc, San Jose, CA, USA). Friedman repeated-measures ANOVA on ranks was used to analyze the sequential HOA changes with both lenses. The appropriate post hoc Tukey correction for multiple comparisons was used. Total HOAs, FI, and SI values of the two tested lenses were compared with each other using a Wilcoxon signed-rank test. The difference between the incidence of fluorescein corneal staining of both CLs was tested for significance using McNemar's test. Alpha level was set at 0.05 for all statistical tests.

\section{Results}

Twenty subjects ( 1 man and 19 women; age, $28.4 \pm 4.5$ years) were enrolled in this study. The mean sphere was $-3.95 \pm 1.84$ $\mathrm{D}$ and the mean cylinder was $-0.44 \pm 0.27 \mathrm{D}$. Log MAR best CL-corrected visual acuity were $-0.15 \pm 0.08$ (senofilcon A lenses) and $-0.14 \pm 0.08$ (senofilcon A lenses). There were no dropout subjects. Significant changes in total HOAs over time were found with etafilcon A lenses $(p<0.01)$, but not with senofilcon A lenses during the postblink 10-s test ( $p=0.348$ ). At eight, nine, and $10 \mathrm{~s}$ after the blink, the total HOAs with etafilcon A lenses were significantly higher than total HOAs at 1 and $2 \mathrm{~s}$ after the blink $(p<0.05)$. Mean total HOAs, FI, and SI values calculated for the two lenses are shown in Table 2 . Significantly $(p<0.001)$ lower total HOAs were obtained with senofilcon A lenses than with etafilcon A lenses. Both FI and SI were significantly lower with senofilcon A lenses than with etafilcon A lenses $(p=0.001$, $p=0.007$ ).

Figure 1 shows the sequential changes in the colorcoded map of ocular total HOAs obtained during the postblink $10 \mathrm{~s}$ coupled with changes in the simulated retinal images of a Landolt ring. While deterioration of the retinal images was notable after the blink with the etafilcon A lens, the postblink retinal images were clearly defined and stable with senofilcon A lens.

On slit-lamp examination, immediately after CL removal following 1-week of wear, smile pattern of corneal staining was observed in eight subjects $(40 \%)$ with etafilcon A lenses and in two subjects (10\%) with senofilcon A lenses $(p=0.041)$. In these two subjects, corneal staining was also observed with etafilcon A lenses. The smile pattern in inferior corneal epithelial staining was observed in six subjects wearing etafilcon A lens, but was not seen in subjects wearing senofilcon A lens.

\section{Discussion}

Safety, comfort, and good visual performance should be prioritized for CL wear. CL manufacturers make enormous efforts to achieve safety and comfort in CL wear. However, because most of the CL users, who have refractive errors without ocular surface disease, generally achieve satisfactory visual acuity, manufactures have placed less emphasis on improving visual performance compared to other qualities of CLs. In the past, the lack of attention to visual performance was perhaps also partly due to the difficulty in evaluating detailed on-eye visual performance or optical quality of CLs, which were only

Table 2 Comparison Of Wavefront Data Between Etafilcon A Lens And Senofilcon A Lens

\begin{tabular}{|l|l|l|l|}
\hline Wavefront & Etafilcon A Lens & Senofilcon A Lens & P-value \\
\hline Total HOAs $(\mu \mathrm{m})$ & $0.168 \pm 0.049$ & $0.131 \pm 0.044$ & $<0.001$ \\
Fluctuation index of total HOAs & $0.034 \pm 0.034$ & $0.013 \pm 0.008$ & 0.001 \\
Stability index of total HOAs & $0.008 \pm 0.011$ & $0.001 \pm 0.003$ & 0.007 \\
\hline
\end{tabular}

Abbreviation: HOA, higher-order aberrations. 


\section{etafilcon A lens}

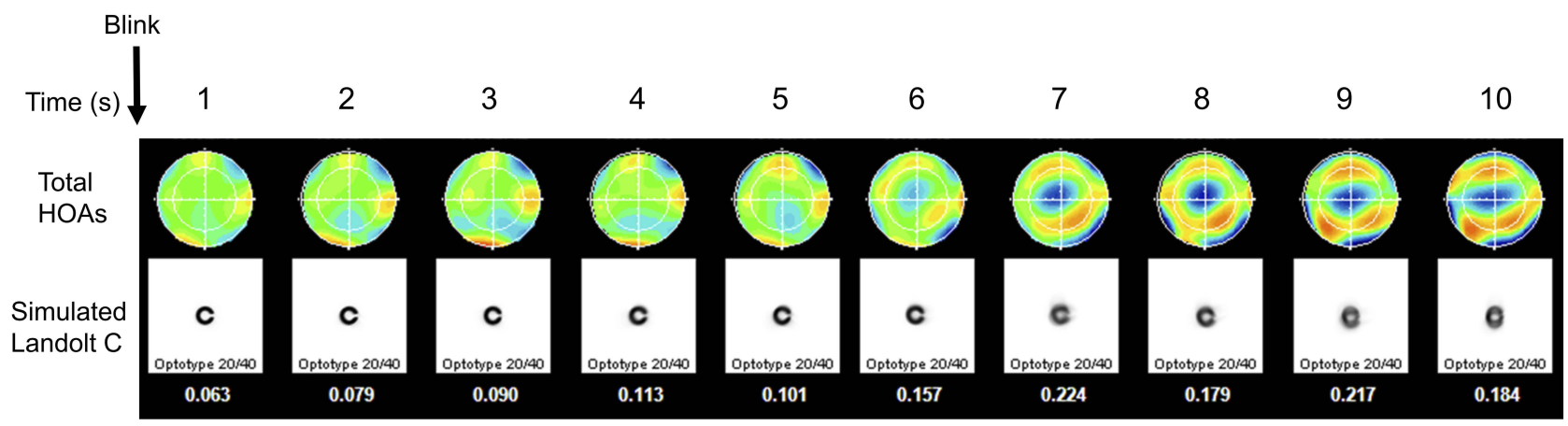

\section{senofilcon A lens}

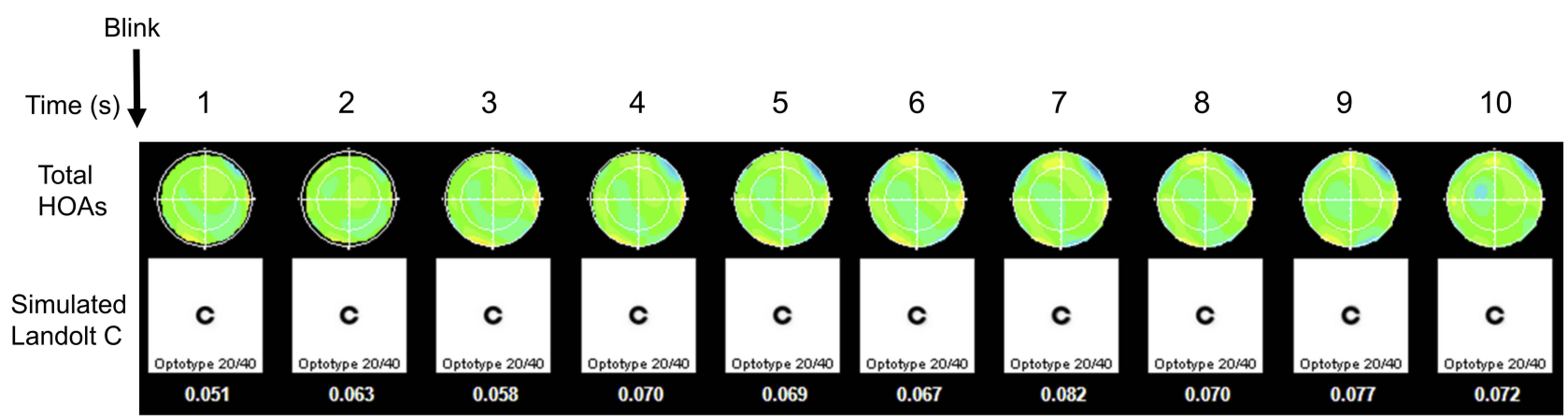

Figure I Sequential wavefront, color-coded maps of ocular higher-order aberrations with etafilcon A lens and senofilcon A lens in a 23-year-old woman. The simulated retinal images of a Landolt ring are shown.

Abbreviation: Total HOAs, total higher-order aberrations.

achieved using standard visual acuity. SCL wearers often complain about blurring and fluctuating vision associated with eye dryness. ${ }^{6}$ In addition, a recent Japanese study reported that approximately $75 \%$ of Japanese SCL wearers experienced changeable, blurry vision. ${ }^{17}$

Previously, we utilized sequential measurements of HOAs and evaluated the effect of internal wetting agents added to hydrogel $\mathrm{CLs}^{7}$ and material of CLs on HOAs. ${ }^{18}$ In the current study, our results demonstrated that daily disposable SiHy CL with high molecular weight polyvinyl pyrrolidone enhanced the visual performance of CL wear during prolonged periods compared to a hydrogel lens without the incorporation of polyvinyl pyrrolidone as a wetting agent. If the average HOA values between eyeblinks are compared, the difference between the two lenses would not be clinically significant. However, the differences in FI and SI, reflecting the alternations of HOAs over a period of time, were remarkable. These results revealed that daily disposable senofilcon A lenses could maintain stable surface lens wettability, and thus, stable visual performance under prolonged gazing conditions. In previous studies, ${ }^{7,18}$ measurements were performed after only $1 \mathrm{hr}$ of CL wear despite the fact that symptoms of eye dryness worsened towards the end of the day. ${ }^{19}$ Therefore, in the current study, we measured on-eye optical quality of SiHy daily disposable CLs closely at the end of the day after a 1-week use of the CLs, thus evaluating the visual performance in conditions similar to daily life. The advantage of senofilcon A lens with the internal wetting agent over the etafilcon A lens without the wetting agent on the ocular surface was also shown by the significantly lower incidence of smile pattern corneal staining, ${ }^{20}$ a typical sign of desiccation. Further investigation of associated factors such as type of work the subjects performed and time looking at screens would be of interest.

The majority of recent-generation SiHy CLs contain modifications to improve comfort primarily by using extra wetting technology, such as the plasma coating, water gradient technology, and internal wetting agent. Previously, Montés-Micó et $\mathrm{al}^{14}$ quantified the diurnal 
variations in optical quality among different daily disposable CLs including recent-generation SiHy CLs. According to their study ${ }^{14}$ the daily disposable SiHy CL with water gradient technology showed low HOA values both at baseline and at the end of the day, while the other two daily disposable SiHy CLs showed poorer performance than daily disposable hydrogel CLs. By comparing the visual performance by measuring visual acuity and contrast sensitivity, Montés-Micó et al ${ }^{14}$ also concluded that differences in visual function could be attributed to inherent CL properties. ${ }^{21}$ In our study, we did not investigate the optical properties of the two used CLs themselves. According to the manufacturer, optical designs of the two products are the same, with both having spherical front and back surface curvatures. The cylinder of each subject in the present study was lower than $0.75 \mathrm{D}$; however, we cannot rule out the effect of astigmatism on HOAs. In future studies, factors such as the optical properties of the lens or refraction in subjects should be considered.

It has been reported that refitting with the 2-week senofilcon A lens alleviated dryness and improved comfort than habitual CLs. ${ }^{22}$ Also, wearing the 2-week type senofilcon A lens provided significantly lower discomfort than wearing habitual CLs or not wearing any CLs in a controlled adverse environment. ${ }^{23}$ According to the manufacturer, the daily disposable senofilcon A lens uses an enhanced moisture network that increases the crosslinking density of the silicone and incorporates a long-chain, high-molecular weight form of polyvinyl pyrrolidone as an internal wetting agent. ${ }^{24}$ In the modern information technology era, many individuals wearing CLs are exposed to the prolonged, everyday use of digital devices and challenging environments such as air conditioning and dirty or polluted air. The 10-s blink interval used in our wavefront measurement overlapped with the blink rate during work on a video display terminal. ${ }^{25,26}$ Therefore, our results suggest that daily disposable senofilcon A lens may be one of the best options for those seeking stable visual performance with the heavy digital device usage and challenging environments.

Inferior corneal epithelial staining was observed in six subjects wearing etafilcon A lens but was not seen in those wearing senofilcon A lens. Due to concerns that CL removal could potentially impact tear film stability, we did not measure the fluorescein tear film break-up time just after the CL removal at the visit following 1 week of CL wear. In our clinical practice, we have experienced some cases where mild dry eye was improved by switching to tested daily disposable senofilcon A lens. Although the lack of assessment of the status of dry eyes and subjective symptoms was a weakness of this study, our results suggest that senofilcon A lens may at least reduce worsening or even improve signs of dry eyes. The characteristics of a CL that is most suited for a patient experiencing dry eye symptoms are as follows: a lens that is highly lubricious (low friction), a lens with minimal in-eye dehydration, and a daily disposable lens. ${ }^{27}$ It would be interesting to assess whether the daily disposable senofilcon A lens, which fulfils these characteristics, would be suited for prescription to mild dry eye patients owing to its superiority in safety, comfort, and visual performance.

This study has some limitations. The lack of data regarding dry eye status and subjective symptoms at enrollment and after 1 week of wear is a weakness of this study. It was desirable to ask the subjects to make a visit without CLs in advance and to assess the tear break-up time, and conduct the validated dry eye questionnaire and meibography. As previous studies have shown that the Contact Lens Dry Eye Questionnaire-8 (CLDEQ-8) is capable of reflecting an improvement or the worsening of Overall Opinion after refitting new SCLs, ${ }^{28,29}$ comparison between changes in these subjective scores, and objectively measured wavefront data before and after refitting the daily disposable senofilcon A lens would be of interest. We confirmed that all subjects adhered to the use of both CLs for over $12 \mathrm{hrs;} \mathrm{however,} \mathrm{we}$ did not ask them to record the number of hours of daily wear of each lens. Collection of this data would have allowed us to determine if the two types of CLs were worn for equal durations daily, to make a fair comparison. Between the use of the two lens types, there should have been a wash out period, during which the subjects are not wearing any lens. Since corneal staining was mild, we thought that it would not preclude CL wear. In addition, we did not perform sample size calculation in the study. The appropriate sample size should have been calculated. The unbalanced male-to-female ratio in this study also needs improvement in a future study with more subjects.

In conclusion, quantitative sequential measurement of HOAs showed that SiHy daily disposable CL with internal wetting agent may yield better and more stable optical quality with less incidence of corneal staining compared to hydrogel lens without the added wetting 
agent, possibly due to a novel internal wetting technology. As reported previously, ${ }^{5}$ the choice of material, SiHy or hydrogel for the daily disposable modality, may be based on patient and practitioner preference. We believe that our study may add new insights into selection of CLs from the viewpoint of visual performance in addition to safety and comfort.

\section{Abbreviations}

CL, contact lens; FI, fluctuation index; HOAs, higherorder aberrations; SCL, soft contact lens; SiHy, silicone hydrogel; SI, stability index.

\section{Ethical Approval And Informed Consent}

This prospective study was reviewed and approved by the institutional review board of Osaka University Hospital. The study adhered to the tenets of the Declaration of Helsinki. All participants provided written informed consent after receiving an explanation on the nature and possible consequences of the study.

\section{Author Contributions}

All authors contributed to data analysis, drafting and revising the article, gave final approval of the version to be published, and agree to be accountable for all aspects of the work.

\section{Disclosure}

Dr Koh has had research supported in the past by Johnson \& Johnson Vision Care, Inc. The authors report no other conflicts of interest in this work.

\section{References}

1. Dumbleton K. Adverse events with silicone hydrogel continuous wear. Cont Lens Anterior Eye. 2002;25:137-146. doi:10.1016/S1367-0484(02) 00009-7

2. Sweeney D, Du Toit R, Keay L, et al. Clinical performance of silicone hydrogel lenses. In: Sweeney D, editor. Silicone Hydrogels: Continuous Wear Contact Lenses. 2nd ed. Oxford: Butterworth-Heinemann; 2004:164-216.

3. Stapleton F, Stretton S, Papas E, Skotnitsky C, Sweeney DF. Silicone hydrogel contact lenses and the ocular surface. Ocul Surf. 2006;4:2443. doi:10.1016/S1542-0124(12)70262-8

4. Chalmers RL, Hickson-Curran SB, Keay L, Gleason WJ, Albright R. Rates of adverse events with hydrogel and silicone hydrogel daily disposable lenses in a large postmarket surveillance registry: the TEMPO registry. Invest Ophthalmol Vis Sci. 2015;56(1):654-663. doi:10.1167/iovs.14-15582

5. Diec J, Tilia D, Thomas V. Comparison of silicone hydrogel and hydrogel daily disposable contact lenses. Eye Contact Lens. 2018;44 (Suppl 1):S167-S172. doi:10.1097/ICL.0000000000000363
6. Begley CG, Chalmers RL, Mitchell GL, et al. Characterization of ocular surface symptoms from optometric practices in North America. Cornea. 2001;20(6):610-618. doi:10.1097/00003226-2001 08000-00011

7. Koh S, Maeda N, Hamano T, et al. Effect of internal lubricating agents of disposable soft contact lenses on higher-order aberrations after blinking. Eye Contact Lens. 2008;34(2):100-105. doi:10.1097/ ICL.0b013e31812e008b

8. Szczesna-Iskander DH, Iskander DR, Read SA, Alonso-Caneiro D. Noninvasive in vivo assessment of soft contact lens type on tear film surface quality. Invest Ophthalmol Vis Sci. 2012;53(1):525-531. doi:1 0.1167/iovs.11-8257

9. Varikooty J, Keir N, Richter D, Jones LW, Woods C, Fonn D. Comfort response of three silicone hydrogel daily disposable contact lenses. Optom Vis Sci. 2013;90(9):945-953. doi:10.1097/ OPX.0b013e31829d8dbf

10. Morgan PB, Chamberlain P, Moody K, Maldonado-Codina C. Ocular physiology and comfort in neophyte subjects fitted with daily disposable silicone hydrogel contact lenses. Cont Lens Anterior Eye. 2013;36(3):118-125. doi:10.1016/j.clae.2012.12.001

11. Schafer J, Steffen R, Reindel W, Chinn J. Evaluation of surface water characteristics of novel daily disposable contact lens materials, using refractive index shifts after wear. Clin Ophthalmol. 2013;54(15): 1973-1979.

12. Wolffsohn JS, Mroczkowska S, Hunt OA, Bilkhu P, Drew T, Sheppard A. Crossover evaluation of silicone hydrogel daily disposable contact lenses. Optom Vis Sci. 2015;92(11):1063-1068. doi:10. 1097/OPX.0000000000000706

13. Varikooty J, Schulze MM, Dumbleton K, et al. Clinical performance of three silicone hydrogel daily disposable lenses. Optom Vis Sci. 2015;92(3):301-311. doi:10.1097/OPX.0000000000000514

14. Montés-Micó R, Belda-Salmerón L, Ferrer-Blasco T, Albarrán-Diego C, García-Lázaro S. On-eye optical quality of daily disposable contact lenses for different wearing times. Ophthalmic Physiol Opt. 2013;33(5):581-591. doi:10.1111/opo.12044

15. Martinez CE, Applegate RA, Klyce SD, McDonald MB, Medina JP, Howland HC. Effect of pupillary dilation on corneal optical aberrations after photorefractive keratectomy. Arch Ophthalmol. 1998;116 (8):1053-1062. doi:10.1001/archopht.116.8.1053

16. Koh S, Maeda N, Hirohara Y, et al. Serial measurements of higherorder aberrations after blinking in normal subjects. Invest Ophthalmol Vis Sci. 2006;47(8):3318-3324. doi:10.1167/iovs.06-0018

17. Koh S, Chalmers R, Kabata D, Shintani A, Nishida K . Translation and validation of the 8-item Contact Lens Dry Eye Questionnaire (CLDEQ-8) among Japanese soft contact lens wearers: the J-CLDEQ-8. Cont Lens Anterior Eye.2019;42:533-539. doi:10.1016/ j.clae.2019.03.002. [Epub ahead of print].

18. Koh S, Higashiura R, Maeda N. Overview of objective methods for assessing dynamic changes in optical quality. Eye Contact Lens. 2016;42(5):333-338. doi:10.1097/ICL.0000000000000232

19. Nichols KK, Redfern RL, Jacob JT, et al. The TFOS International Workshop on Contact Lens Discomfort: report of the definition and classification subcommittee. Invest Ophthalmol Vis Sci. 2013;54(11): TFOS14-19. doi:10.1167/iovs.13-13074

20. Watanabe K, Hamano $H$. The typical pattern of superficial punctate keratopathy in wearers of extended wear disposable contact lenses. Clao J. 1997;23(2):134-137.

21. Belda-Salmerón L, Ferrer-Blasco T, Albarrán-Diego C, Madrid-Costa D, Montés-Micó R. Diurnal variations in visual performance for disposable contact lenses. Optom Vis Sci. 2013;90(7):682-690. doi:10.1097/OPX.0b013e318299088f

22. Riley C, Young G, Chalmers R. Prevalence of ocular surface symptoms, signs, and uncomfortable hours of wear in contact lens wearers: the effect of refitting with daily-wear silicone hydrogel lenses (senofilcon a). Eye Contact Lens. 2006;32(6):281-286. doi:10.1097/01. icl.0000224522.04723.7a 
23. Ousler GW, Anderson RT, Osborn KE. The effect of senofilcon A contact lenses compared to habitual contact lenses on ocular discomfort during exposure to a controlled adverse environment. Curr Med Res. 2007;24(2):335-341. doi:10.1185/030079908X260826

24. Keir N, Jones L. Wettability and silicone hydrogel lenses: a review. Eye Contact Lens. 2013;39(1):100-108. doi:10.1097/ICL.0b013e31827d546e

25. Tsubota K, Nakamori K. Dry eyes and video display terminals. $N$ Engl J Med. 1993;328(8):584. doi:10.1056/NEJM199302253280817

26. Freudenthaler N, Neuf H, Kadner G, Schlote T. Characteristics of spontaneous eyeblink activity during video display terminal use in healthy volunteers. Graefes Arch Clin Exp Ophthalmol. 2003;241 (11):914-920. doi:10.1007/s00417-003-0786-6
27. Efron N. Complications. In: Efron N, editor. Contact Lens Practice. 3rd ed. New York: Elsevier; 2014:384-409.

28. Chalmers RL, Begley CG, Moody K, Hickson-Curran SB. Contact lens dry eye questionnaire-8 (CLDEQ-8) and overall opinion of contact lens performance. Optom Vis Sci. 2012;89(10):1435-1442. doi:10.1097/OPX.0b013e318269c90d

29. Chalmers RL, Keay L, Hickson-Curran SB, Gleason WJ. Cutoff score and responsiveness of the 8-item Contact Lens Dry Eye Questionnaire (CLDEQ-8) in a Large daily disposable contact lens registry. Cont Lens Anterior Eye. 2016;39(5):342-352. doi:10.1016/j. clae.2016.04.005
Clinical Ophthalmology

\section{Publish your work in this journal}

Clinical Ophthalmology is an international, peer-reviewed journal covering all subspecialties within ophthalmology. Key topics include: Optometry; Visual science; Pharmacology and drug therapy in eye diseases; Basic Sciences; Primary and Secondary eye care; Patient Safety and Quality of Care Improvements. This journal is indexed on PubMed

Submit your manuscript here: https://www.dovepress.com/clinical-ophthalmology-journal
Dovepress

Central and CAS, and is the official journal of The Society of Clinical Ophthalmology (SCO). The manuscript management system is completely online and includes a very quick and fair peer-review system, which is all easy to use. Visit http://www.dovepress.com/ testimonials.php to read real quotes from published authors. 